\title{
THE ITERATED EQUATION OF GENERALIZED AXIALLY SYMMETRIC POTENTIAL THEORY, VI
}

\author{
GENERAL SOLUTIONS \\ J. C. BURNS \\ (Received 24 November 1972) \\ Communicated by A. F. Pillow
}

\section{Introduction}

The iterated equation of generalized axially symmetric potential theory [1] is the equation

$$
L_{k}^{n}(f)=0 \text {, }
$$

where, in its simplest form, the operator $L_{k}$ is defined by

$$
L_{k}(f) \equiv \partial^{2} f / \partial x^{2}+\partial^{2} f / \partial y^{2}+k y^{-1} \partial f / \partial y,
$$

the function $f \equiv f(x, y)$ being assumed to belong to the class of $C^{2 n}$ functions and the parameter $k$ to take any real value. In appropriate circumstances, which will be indicated later, the operator can be generalized but as this can be done without altering the methods used, the operator will be taken in the form (2) or in the corresponding form

$$
L_{k}(f) \equiv \frac{\partial^{2} f}{\partial r^{2}}+\frac{1+k}{r} \frac{\partial f}{\partial r}+\frac{1-\mu^{2}}{r^{2}} \frac{\partial^{2} f}{\partial \mu^{2}}-\frac{(1+k) \mu}{r^{2}} \frac{\partial f}{\partial \mu},
$$

where $r, \theta$ are polar coordinates such that $x=r \cos \theta, y=r \sin \theta$ and $\mu \equiv \cos \theta$.

It is known that equation (1) has many particular solutions which involve solutions of the simple equation

$$
L_{k}(f)=0
$$

or of similar equations with a different value of the parameter $k$. (Solutions of equation (4) will be denoted by $f_{k}$ and solutions of equation (1), for $n>1$, by $f_{k}^{(n)}$.) Thus, for example, it is shown in [2] that each member of each of the following sets of terms, defined for $0 \leqq s \leqq n-1$, is a solution of (1):

(i) $f_{k-2 s}$

(ii) $y^{2 s} f_{k+2 s}$

(iii) $x^{s} f_{k}$

(iv) $r^{2 s} f_{k}$. 
It follows that all expressions of the form

(i) $\sum_{s=0}^{n-1} f_{k-2 s} \quad$ (ii) $\sum_{s=0}^{n-1} y^{2 s} f_{k+2 s}$

(iii) $\sum_{s=0}^{n-1} x^{s} f_{k, s}$

(iv) $\sum_{s=0}^{n+1} r^{2 s} f_{k, s}$

are solutions of the iterated equation (1). (The notation $\left\{f_{k, s}\right\}$ is used to denote a set of solutions of (4).) It is natural to ask whether these sums of $n$ particular solutions are general solutions of equation (1); that is, to ask whether any solution of the equation can be expressed in the given form.

In fact, all four of the sums in (6) are known to be general solutions of (1). Weinstein [3] showed that (6) (i) is a general solution while Payne [4] proved the result for (6) (ii). In the same paper, Payne remarks that it is well-known that (6) (iii) and (6) (iv) are general solutions of (1).

The terms which make up the sum in (6) (ii) (and also those in (6) (i)) are of the form $y^{s} f_{t}$ and there are many other terms of this form which are solutions of (1). The complete set of such solutions has been found [2] and a criterion has been given by which it can be decided whether the sum of any particular set of $n$ terms chosen from this set forms a general solution [5]. The discussion of this problem starts with Weinstein's general solution (6) (i) and relates other solutions to this. It turns out that there are many general solutions of (1) given by the sum of $n$ terms of the form $y^{s} f_{t}$, among them both (6) (ii) and (6) (i).

In this paper, a similar discussion is given of general solutions of (1) made up of the sum of $n$ terms chosen from sets of particular solutions of the form $x^{s} f_{t}$ or $r^{2 s} f_{t}$. The first step is to find the complete sets of solutions of these kinds. Then it is shown that, in particular, (6) (iii) and (6) (iv) are general solutions. Although these results are not new, proofs are given for them as they are necessary in the argument. Moreover, in each case, the method of proof given here is essentially that used by Weinstein [3] in establishing that (6) (i) is a general solution and it seems worth while to demonstrate the unified approach to these problems made possible by the use of this method. Finally, a criterion is given by which it can be decided that the sum of $n$ given terms of each of the forms under discussion constitutes a general solution of (1). The procedure is again based on the recognition that in each case the terms of Weinstein's solution (6) (i) are included in the family of particular solutions so that the sums of $n$ terms being discussed can be related to Weinstein's solutions and in this way shown to be general solutions. In each case there are many general solutions of the given form including (6) (iii) and (6) (iv) respectively (and (6) (i) appears as a specially simple member of each set of general solutions).

\section{Solutions of $L_{k}^{n}(f)=0$ of the form $x^{s} f_{t}$}

In [2] it is shown that, for any function $f$ and any integers $n, s$ such that $n \geqq s \geqq 1$, 


$$
L_{k}^{n}\left(x^{s} f\right)=\mathscr{L}_{n, k} \mathscr{L}_{n-1, k}, \cdots, \mathscr{L}_{n-s+1, k} L_{k}^{n-s}(f),
$$

where $\mathscr{L}_{n, k} \equiv x L_{k}+2 n \partial / \partial x$. It is further shown in [2] that

$$
L_{k}^{n}\left(f_{l}\right)=(k-l-2)(k-l-4) \cdots[k-l-2(n-1)] \mathscr{D}^{n}\left(f_{l}\right)
$$

where the operator $\mathscr{D} \equiv y^{-1} \partial / \partial y$.

From (7) and (8) (and the condition $L_{k}\left(f_{k}\right)=0$ ), it follows that $L_{k}^{n}\left(x^{s} f_{k-2 t}\right)=0$ if and only if $s$ and $t$ are non-negative integers such that $0 \leqq s+t$ $\leqq n-1$. Thus there is a set of $\frac{1}{2} n(n+1)$ particular solutions of (1) which can conveniently be denoted by $x^{s} f_{k-2 t, s}$ and the problem now is to decide whether the sum of a given set of terms chosen from this set can form a general solution of (1).

$$
\text { 3. General solution of } L_{k}^{n}(f)=0 \text { of form } \sum_{s=0}^{n-1} x^{s} f_{k, s}
$$

3.1 The first step in finding all general solutions of (1) which consist of the sum of $n$ terms chosen from the family of solutions of the form $x^{s} f_{k-2 t, s}$ given in section 2 is to show that the particular case of the sum of the $n$ terms of this family for which $t=0$ is a general solution. This is the sum, $\sum_{s=0}^{n-1} x^{s} f_{k, s}$, given in (6) (iii).

3.2 From section 2 it is clear that every function of the form $\sum_{s=0}^{n-1} x^{s} f_{k, s}$ is a solution of (1). It must now be proved that every solution of (1) can be expressed as a sum of terms of this kind.

As so frequently happens, it pays to look first at the simplest case and to prove that every solution of the equation

$$
L_{k}^{2}(f)=0
$$

can be expressed in the form $f_{k, 0}+x f_{k, 1}$.

Equation (9) is equivalent to the equation

$$
L_{k}(f)=f_{k}
$$

where $f_{k}$ is an arbitrary solution of (4); for it is clear that every solution of (10) for any function $f_{k}$ is a solution of (9) and every solution of (9) satisfies (10) with some $f_{k}$ on the right hand side.

If a function $f_{k, 1}$, a solution of (4), could be found such that $f_{k}=L_{k}\left(x f_{k, 1}\right)$, equation (10) would become $L_{k}\left(f-x f_{k, 1}\right)=0$ which clearly has general solution of the form $f=f_{k, 0}+x f_{k, 1}$ as required. Since, as is easily verified, $L_{k}\left(x f_{k, 1}\right)$ $=2 \partial f_{k, 1} / \partial x$, it is now necessary only to show that when $f_{k}$ is given, it is possible to find a function $f_{k, 1}$ such that $f_{k}=2 \partial f_{k, 1} / \partial x$.

With a slight change of notation, the problem is expressed more conveniently thus: given a function $f_{k}$, which is a solution of (4), show how to find a function $F$ such that (i) $\partial F / \partial x=f_{k}$ (ii) $L_{k}(F)=0$. 
Condition (i) is satisfied by choosing

$$
F(x, y)=\int_{b}^{x} f_{k}(\xi, y) d \xi+\phi(y, b)
$$

where $b$ is a constant.

It will be possible to find this function $F(x, y)$ provided condition (ii) leads to an ordinary differential equation for $\phi(y, b)$ as a function of $y$. The condition $L_{k}(f)=0$ gives

$$
\frac{\partial f_{k}}{\partial x}(x, y)+\int_{b}^{x}\left\{\frac{\partial^{2} f_{k}}{\partial y^{2}}(\xi, y)+\frac{k}{y} \frac{\partial f_{k}}{\partial y}(\xi, y)\right\} d \xi+\frac{d^{2} \phi}{d y^{2}}(y, b)+\frac{k}{y} \frac{d \phi}{d y}(y, b)=0 .
$$

Since $L_{k}\left(f_{k}\right)=0$, the integrand in the second term is equal to $-\partial^{2} f_{k}(\xi, y) / \partial \xi^{2}$ so the integral is easily evaluated. The result is an ordinary differential equation for $\phi(y, b)$ :

$$
\frac{d^{2} \phi}{d y^{2}}(y, b)+\frac{k}{y} \frac{d \phi}{d y}(y, b)+\frac{\partial f_{k}}{\partial x}(b, y)=0 .
$$

This equation can be solved for $\phi(y, b)$ so the required function $F(x, y)$ can be obtained.

3.3. Now that it has been shown that, when $n=2$, the equation $L_{k}^{n}(f)=0$ has a general solution of the form $\sum_{s=0}^{n-1} x^{s} f_{k, s}$, mathematical induction can be used to show that the result holds generally.

The proof makes use of Weinstein's general solution already referred to: any solution of (1) can be expressed in the form $\sum_{s=0}^{n-1} f_{k-2 s}$. Because the required general solution has been shown to exist when $n=2$, it follows that

$$
f_{k, 0}+x f_{k, 1} \leftrightarrow f_{k}+f_{k-2}
$$

where the symbol $\leftrightarrow$ is used to mean that each side can be expressed in the form of the other. If now it is assumed that the result holds for the equation $L_{k}^{n}(f)=0$ for any integer $n$, it is similarly true that

$$
\sum_{s=0}^{n-1} x^{s} f_{k s} \leftrightarrow \sum_{s=0}^{n-1} f_{k-2 s}
$$

To complete the inductive argument, it is sufficient to prove that (13) still holds when the upper limit of the two sums is replaced by $n$.

Since $\sum_{s=0}^{n} x^{s} f_{k, s}=f_{k, 0}+x \sum_{s=0}^{n-1} x^{s} f_{k, s+1}$, it follows from (13) that, for some functions $F_{k-2 s}$,

$$
\begin{aligned}
\sum_{s=0}^{n} x^{s} f_{k, s} & \leftrightarrow f_{k, 0}+x \sum_{s=0}^{n-1} F_{k-2 s} \\
& \leftrightarrow\left(f_{k 0}+x F_{k}\right)+x \sum_{s=1}^{n-1} F_{k-2 s} .
\end{aligned}
$$


Because of (12), this gives

$$
\begin{aligned}
\sum_{s=0}^{n} x f_{k s} & \leftrightarrow f_{k}+f_{k-2}+x \sum_{s=1}^{n-1} F_{k-2 s} \\
& =f_{k}+\left(f_{k-2}+x F_{k-2}\right)+x \sum_{s=2}^{n-1} F_{k-2 s}
\end{aligned}
$$

Once again (12) is applied and the process is repeated until the stage is reached where

$$
\sum_{s=0}^{n} x^{s} f_{k, s} \leftrightarrow \sum_{s=0}^{n-2} f_{k-2 s}+\left(f_{k-2(n-1)}+x F_{k-2(n-1)}\right)
$$

A final application of (12) then gives the required result.

4. General solutions of $L_{k}^{n}(f)=0$ made up of the sum of $n$ terms

$$
\text { of the form } x^{s} f_{k-2 t, s}
$$

It has been seen that $x^{s} f_{k-2 t, s}$ is a solution of (1) provided it is one of the $\frac{1}{2} n(n+1)$ terms for which the non-negative integers $s, t$ satisfy the conditions $0 \leqq s+t \leqq n-1$. It is convenient to think of this set of solutions as represented by the points of the triangular array in the $(s, t)$-plane as shown in figure 1(a) for the case $n=4$.

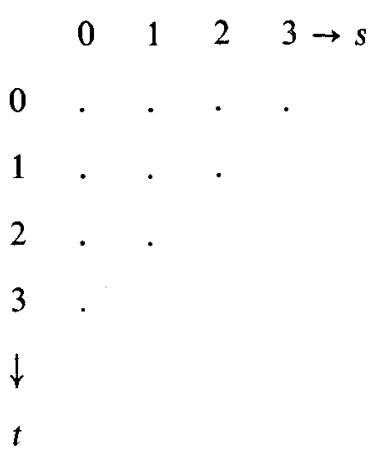

(a) (b)

(c)

Figure 1.

The general solution obtained in section 3 is the sum of the $n$ terms for which $t=0$ and these are represented by the points of the top row of the array as indicated in figure 1(b). There is one of these points in each of the diagonal rows of the array for which $s+t=N, 0 \leqq N \leqq n-1$.

It will now be proved that the sum of any set of $n$ terms represented by points of this array which lie one in each diagonal row (e.g. the one shown in 
figure 1(c)) is a general solution of equation (1). Clearly there will be $n$ ! such sets of points and corresponding general solutions.

The result is clearly true when $n=1$ and $n=2$ and it is proved generally by mathematical induction. For this purpose it is sufficient to prove that if the equations $L_{k}^{m}(f)=0$ for $2 \leqq m \leqq n$ have general solutions of the required form, then so also does the equation $L_{k}^{n+1}(f)=0$.

Because of the inductive hypothesis, any set of $n+1$ terms satisfying the condition for selection from the solutions of $L_{k}^{n+1}(f)=0$ of the form $x^{s} f_{k-2 t, s}$, $0 \leqq s+t \leqq n$, can be expressed in the form $f_{k}^{(n)}+x^{n-p} f_{k-2 p, n-p}$ where $L_{k}^{n}\left(f_{k}^{(n)}\right)=0$ and $0 \leqq p \leqq n$. (The term $x^{n-p} f_{k-2 p . n-p}$ is represented by the point $(n-p, p)$ on the longest diagonal row $s+t=n$ of the triangle of points in the $(s, t)$-plane representing the family of solutions of the equation $L_{k}^{(n+1)}(f)=0$.) The function $f_{k}^{(n)}$ can be replaced by any of the assumed general solutions of the equation $L_{k}^{(n)}(f)=0$ and the one to choose is

$$
\sum_{t=0}^{p-1} f_{k-2 t .0}+\sum_{s=0}^{n-p-1} x^{s} f_{k-2 p s}
$$

(In the case $p=0$, the first sum is to be taken as zero and when $p=n$ the second sum is to be taken as zero.) The original sum of $n+1$ particular solutions of $L_{k}^{n+1}(f)=0$ is thus equivalent to

$$
\sum_{t=0}^{p-1} f_{k-2 t, 0}+\sum_{s=0}^{n-p} x^{s} f_{k-2 p, s} .
$$

The terms in (14) are shown in figure 2(a) for the case $n=5, p=3$.

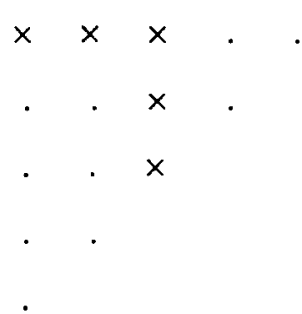

(a) (b)

Figure 2

From Weinstein's general solution (6) (i) and the general solution established in section 3 , it is known that for $0 \leqq p \leqq n$.

$$
\sum_{s=0}^{n-p} x^{s} f_{k, s} \leftrightarrow \sum_{s=0}^{n-p} f_{k-2 s, 0},
$$

both sums being general solutions of the equation $L_{k}^{n-p}(f)=0$. Replacing $k$ by $k-2 p$ in (15) gives an equivalence which is used to convert (14) into 


$$
\sum_{t=0}^{p-1} f_{k-2 t, 0}+\sum_{s=0}^{n-p} f_{k+2 p-2 s, 0}=\sum_{t=0}^{n} f_{k-2 t, 0},
$$

the sum of the terms illustrated in figure 2(b), which, from Weinstein's solution, is seen to be a general solution of the equation $L_{k}^{n+1}(f)=0$. This completes the proof.

\section{Solutions of $L_{k}^{n}(f)=0$ of the form $r^{2 s} f_{t}$}

5.1. The discussion of general solutions of (1) consisting of the sums of $n$ particular solutions of the form $r^{2 s} f_{t}$ follows exactly the pattern developed in sections 2, 3, 4 for general solutions consisting of sums of solutions of the from $x^{s} f_{t}$.

5.2. The first requirement is to find the family of particular solutions of (1) of the form $r^{2 s} f_{t}$. It is shown in [2] that, for any function $f$ and any integers $n, s$ such that $n \geqq s \geqq 1$,

$$
L_{k}^{n}\left(r^{2 s} f\right)=\mathscr{M}_{n, k} \mathscr{M}_{n-1, k} \cdots \mathscr{M}_{n-s+1, k} L_{k}^{n-s}(f),
$$

where $\mathscr{M}_{n, k} \equiv r^{2} L_{k}+4 n r \partial / \partial r+2 n(2 n+k)$.

From (8) and (16) (and the condition $L_{k}\left(f_{k}\right)=0$ ) it follows that $L_{k}^{n}\left(r^{2 s} f_{k-2 t}\right)=0$ if and only if $s$ and $t$ are non-negative integers such that $0 \leqq s+t$ $\leqq n-1$. Again there is a set of $\frac{1}{2} n(n+1)$ particular solutions of (1) which can be denoted by $r^{2 s} f_{k-2 t, s}$ and it remains to consider whether the sum of a given set of $n$ of these particular solutions can form a general solution of (1).

$$
\text { 6. General solution of } L_{k}^{n}(f)=0 \text { of form } \sum_{s=0}^{n-1} r^{s^{2}} f_{k, s}
$$

6.1. As in section 3, the first step in finding all general solutions of (1) made up of the sum of $n$ terms of the form $r^{2 \mathrm{~s}} f_{k-2 t, s}$ given in section 5 is to show that the particular case of the sum of the $n$ terms for which $t=0$ is a general solution. This sum, $\sum_{s=0}^{n-1} r^{2 s} f_{k, s}$, is the one given in (6) (iv).

6.2. From section 5 it is clear that every function of the form $\sum_{s=0}^{n-1} r^{2 s} f_{k s}$ is a solution of (1). As in section 3, it remains to prove that every solution of equation (1) can be expressed in this form.

The simpler equation (9), $L_{k}^{2}(f)=0$, is again considered. As in section 3.2, it is replaced by equation (10) and it is easily seen that if a function $f_{k, 1}$, a solution of (4), can be found such that $f_{k}=L_{k}\left(r^{2} f_{k, 1}\right)$, then a general solution of (9) is given by $f=f_{k, 0}+r^{2} f_{k, 1}$.

With a change of notation, the problem reduces to showing that for a given function $f_{k}$, a function $F_{k}$ can be found such that $f_{k}=L_{k}\left(r^{2} F_{k}\right)$. In this case,

$$
L_{k}\left(r^{2} F_{k}\right)=4 r \partial F_{k} / \partial r+(4+2 k) F_{k}
$$


and, whereas in the previous case the corresponding expression was a simple multiple of $\partial F_{k} / \partial x$, here the right hand side of (17) is not just a multiple of $\partial F_{k} / \partial r$.

The analogy with the previous case suggests that it should be possible at least to solve the simpler problem of finding $\phi_{k}$ so that $f_{k}=r \partial \phi_{k} / \partial r$. This problem is: given a function $f_{k}$, such that $L_{k}\left(f_{k}\right)=0$, find a function $\phi$ such that (i) $r \partial \phi / \partial r=f_{k}$ (ii) $L_{k}(\phi)=0$. It turns out that this problem can be solved in exactly the same way as the problem discussed in section 3 .

This simpler theorem both points the way to the solution of the required result and acts as a model for it. It is easily shown from (17) that

$$
\frac{1}{4} r^{1+k / 2} L_{k}\left(r^{2} F_{k}\right)=r \frac{\partial}{\partial r}\left(r^{1+k / 2} F_{k}\right)
$$

so if new functions $g^{(k)}$ and $G^{(k)}$ are defined by

$$
g^{(k)}=\frac{1}{4} r^{1+k / 2} f_{k}, \quad G^{(k)}=r^{1+k / 2} F_{k},
$$

the problem of finding $F_{k}$ so that $L_{k}\left(r^{2} F_{k}\right)=f_{k}$ becomes that of finding $G^{(k)}$ when $r \partial G^{(k)} / \partial r=g^{(k)}$ and $g^{(k)}$ is given. The problem is now analogous to the simpler one just described but the equation satisfied by $g^{(k)}$ and $G^{(k)}$ has yet to be determined. (These two functions are constructed in the same way from solutions of the equation $L_{k}(f)=0$ so both will satisfy the same differential equation.)

It is known [2] that, for any function $f$ and any $m$,

$$
L_{k}\left(r^{m} f\right)=r^{m} L_{k}(f)+2 m r^{m-1} \partial f / \partial r+m(m+k) r^{m-2} f
$$

and from this, with $m=1+k / 2$, it is easily deduced that the operator $M_{k}$, where

$$
\begin{aligned}
M_{k} & \equiv L_{k}-\frac{2+k}{r} \frac{\partial}{\partial r}+\frac{1-k^{2} / 4}{r^{2}} \\
& \equiv \frac{\partial^{2}}{\partial r^{2}}-\frac{1}{r} \frac{\partial}{\partial r}+\frac{1-k^{2} / 4}{r^{2}}+\frac{1-\mu^{2}}{r^{2}} \frac{\partial^{2}}{\partial \mu^{2}}-\frac{(1+k) \mu}{r^{2}} \frac{\partial}{\partial \mu},
\end{aligned}
$$

is such that

$$
M_{k}\left(r^{1+k, 2} f\right)=r^{1+k / 2} L_{k}(f) .
$$

Thus $M_{k}\left(r^{1+k / 2} f\right)=0$ if and only if $L_{k}(f)=0$ and it follows that the functions $g^{(k)}$ and $G^{(k)}$ are solutions of the equation.

$$
M_{k}(g)=0 .
$$

The problem to be solved now takes the form: given a function $g^{(k)}$, a solution of (18), find a function $G$ such that (i) $r \partial G / \partial r=g^{(k)}$ (ii) $M_{k}(G)=0$.

The analogies between the two problems of this section and between both of them and the problem of section 3 are clear. The details of the calculation required here are exactly like those of section 3 so all that need be said is that 
the function $G$ can indeed be found, the solution depending ultimately on solving the equation which corresponds to equation (11):

$$
\begin{gathered}
\left(1-\mu^{2}\right) \frac{d^{2} \phi}{d \mu^{2}}(\mu, b)-(1+k) \mu \frac{d \phi}{d \mu}(\mu, b)+\left(1-\frac{k^{2}}{4}\right) \phi(\mu, b) \\
=2 g_{k}(b, \mu)-b \frac{\partial g_{k}}{\partial r}(b, \mu) .
\end{gathered}
$$

It follows that the equation $L_{k}^{2}(f)=0$ has a general solution of the form $f_{k, 0}+r^{2} f_{k, 1}$.

6.3. The extension of this result to show that the equation $L_{k}^{n}(f)=0$ has a general solution of the form $\sum_{s=0}^{n-1} r^{2 s} f_{k, s}$ follows by the use of mathematical induction, exactly as in section 3.3 .

\section{General solutions of $L_{k}^{n}(f)=0$ made up of the sum of $n$ terms of the form $r^{2 s} f_{k-2 t}$}

If the representative solution of (1) is changed from $x^{s} f_{k-2 t, s}$ to $r^{2 s} f_{k-2 t, s}$, all the discussion of section 4 can be repeated so that again $n$ ! general solutions can be made up of sums of $n$ terms of the given form.

\section{Other forms for the operator $L_{k}$}

It has now been shown, in [2] and in this paper, that the three families of solutions of the equation $L_{k}^{n}(f)=0$ of the form $y^{s} f_{t}, x^{s} f_{t}$ and $r^{2 s} f_{t}$ all give rise to general solutions of this equation which are made up of the sums of $n$ suitably selected members of one of the families.

So far the operator $L_{k}$ has been taken in the form (2) or in the equivalent polar form (3) but in some cases it is possible to apply the same results to related operators. In considering possible generalizations of $L_{k}$ it is neccessary to preserve the characteristics of the operator which are essential to the argument used in establishing the general solution.

It turns out that, in the case of general solutions based on the family $y^{s} f_{t}$, the operator $L_{k}$ can be replaced by $X+\partial^{2} / \partial y^{2}+k y^{-1} \partial / \partial y$ where $X$ is any linear homogeneous operator independent of $y$.

Similarly, in the case of general solutions based on the family $r^{2 s} f_{t}$, the essential terms in the operator $L_{k}$ are those depending only on $r$ and $L_{k}$ can be replaced by any operator of the form $\partial^{2} / \partial r^{2}+(1+k) r^{-1} \partial / \partial r+r^{-2} \Phi$ where $\Phi$ is any linear homogeneous operator independent of $r$.

In the case of general solutions based on the family $x^{s} f_{t}$ however, only a minor formal change is possible in which $L_{k}$ is taken in the form $A \partial^{2} / \partial x^{2}+\partial^{2} / \partial y^{2}$ $+k y^{-1} \partial / \partial y$ for any constant $A$. It will be noted nowever that this change allows the operator to be hyperbolic. 


\section{References}

[1] A. Weinstein, 'Generalized axially symmetric potential theory', Bull. Amer. Math. Soc. 59 (1953), 20-28.

[2] J. C. Burns, 'The iterated equation of generalized axially symmetric potential theory, I. Particular solutions', J. Austral. Math. Soc. 7 (1967), 263-276.

[3] A. Weinstein, 'On a class of partial differential equations of even order', Ann. Mat. Pura Appl. 39 (1955), 245-254.

[4] L. E. Payne, 'Representation formulas for solutions of a class of partial differential equation', J. Math. and Phys. 38 (1959), 145-149.

[5] J. C. Burns, 'The iterated equation of generalized axially symmetric potential theory, II. General solutions of Weinstein's type', J. Austral. Math. Soc. 7 (1967), 277-289.

Faculty of Military Studies

University of New South Wales

Royal Military College

Duntroon, A.C.T. 2600

Australia 